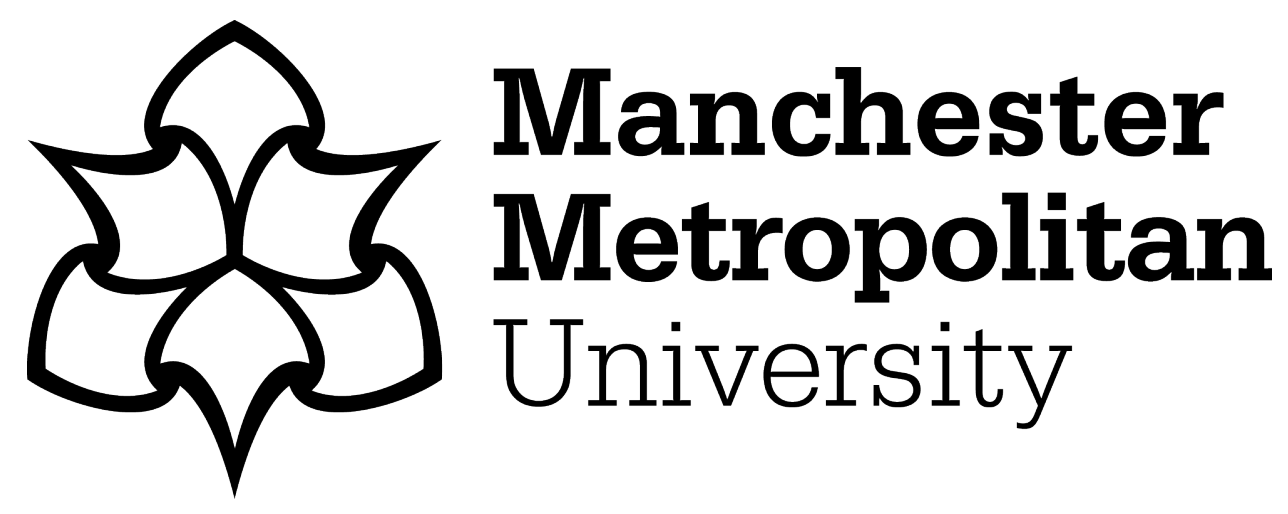

Nema, S and Kowalczyk, P (2016) Detecting Abrupt Changes in a Noisy van der Pol Type Oscillator. Differential Equations and Dynamical Systems, 24 (4). pp. 445-457. ISSN 0971-3514

Downloaded from: https://e-space.mmu.ac.uk/608684/

Version: Accepted Version

Publisher: Springer Verlag

DOI: https://doi.org/10.1007/s12591-015-0252-z

Please cite the published version 


\section{Differential Equations and Dynamical Systems Detecting abrupt changes in a noisy van der Pol type oscillator \\ --Manuscript Draft--}

Manuscript Number:

Full Title:

Article Type:

Corresponding Author:

\section{Corresponding Author Secondary}

Information:

Corresponding Author's Institution:

Manchester Metropolitan University

Corresponding Author's Secondary

Institution:

First Author:

Salam Nema, PhD

First Author Secondary Information:

Order of Authors:

Salam Nema, PhD

Piotr Kowalczyk, PhD

Order of Authors Secondary Information:

Abstract:

Many signals produced by dynamical systems may undergo abrupt changes such as a jump or a sharp change. Detecting such change points is of primary importance in many applications ranging from exploratory data analysis to diagnosis. This paper addresses the detection of abrupt changes in a noisy van der Pol oscillator as a model of an electrical circuit with nonlinear resistance. The proposed approach combines wavelet analysis with information entropy in order to extract signal frequencies corresponding to any abrupt changes that occur. We also investigate the influence of noise intensity on detecting change points in the model system. Performance is evaluated on simulated data generated by using different model parameters.

\section{Suggested Reviewers:}




\title{
Detecting abrupt changes in a noisy van der Pol type oscillator
}

\author{
Salam Nema • Piotr Kowalczyk
}

Received: June 2014

\begin{abstract}
Many signals produced by dynamical systems may undergo abrupt changes such as a jump or a sharp change. Detecting such change points is of primary importance in many applications ranging from exploratory data analysis to diagnosis. This paper addresses the detection of abrupt changes in a noisy van der Pol oscillator as a model of an electrical circuit with nonlinear resistance. The proposed approach combines wavelet analysis with information entropy in order to extract signal frequencies corresponding to any abrupt changes that occur. We also investigate the influence of noise intensity on detecting change points in the model system. Performance is evaluated on simulated data generated by using different model parameters.
\end{abstract}

Keywords Van der Pol, Abrupt change, Oscillator, Wavelet, Instantaneous frequency.

\section{Introduction}

Nonlinear dynamical systems arise in many applications, and are often characterised by the presence of sudden transitions in one or more of their dynamic states. Here we suggest a method for detecting these abrupt changes in systems dynamics. Such systems are often modelled either as a deterministic one [1,2], which ignore the presence of noise, or a stochastic one which, on the other hand, stress randomness $[3,4]$. Deterministic system models with additive noise can be seen as a combination of a deterministic and a stochastic model system, even though formally these are classed as stochastic systems. It is this last class of systems that is of our interest because, practically, all systems are influenced by random movements S. Nema

School of Computing, Mathematics and Digital Technology, Manchester Metropolitan University, Chester Street, Manchester M1 5GD, U.K.

Tel.: +44-161-2471136

E-mail: s.nema@mmu.ac.uk

P. Kowalczyk

School of Computing, Mathematics and Digital Technology, Manchester Metropolitan University, Chester Street, Manchester M1 5GD, U.K.

E-mail: P.Kowalczyk@mmu.ac.uk 
of its intrinsic structural components. For instance, engineering systems which contain switchings and ignitions generate random fluctuations which assist these switchings and ignitions. Also, in electrical conductors there is present a random movement of electrons due to thermal agitation that cannot be eliminated. That is, a system which, for example, switches between different configurations depending on the value of some decision function, and which is additionally affected by noise, would evolve along a trajectory which, if noise was absent, would exhibit a corner, a discontinuous change of curvature or a discontinuity in higher derivative. Obviously, noise will affect such a discontinuity. Depending on the context, it may be of interest to isolate the effects of deterministic mechanism on system's behaviour from that of random fluctuations in order to detect abrupt changes in such hybrid systems.

Detection of change points constitutes a crucial and a challenging problem in different domains. Several methods have been proposed in the literature for different applications to detect change points in the process data. For instance, time-domain methods [5], spectral methods [6], and auto-covariance methods [7]. In particular, Theron and Aldrich [5] developed a time-domain approach for identifying the nonlinearities in dynamic process systems. The idea is to see whether the measured time series, which are similar to the stochastic data, have the same power spectra or autocorrelation functions. Babji et al. [6] proposed a method based on Hilbert-Huang Transform to detect control valve nonlinearity. It was found that the nonlinearity can be captured by Intrinsic Mode Functions obtained from the Empirical Mode Decomposition of the process output. Killick and Eckley [7] introduced a locally stationary wavelet framework to detect changes in general auto-covariance structure within non-stationary time series data.

The character of nonlinearities that we are concerned with here is different from all these cases. We are interested in revealing change points in a deterministic signal buried in white noise where the local stationarity is violated. We consider the problem of how to detect these types of nonlinearties, which we identify by finding sharp changes in the characteristic properties of the signal generated by a stochastic process. We use a stochastic version of van der Pol type oscillator, which possesses a trivial fixed point and a limit cycle attractor. We consider different intensities of white Gaussian noise which might influence the detection of any abrupt changes in the model system. When using traditional methods to reduce noise in time series data, the results cannot meet the practical needs. Hence, we have developed a new methodology aimed to process the time series data based on wavelet decomposition technique. That is, to investigate the 'deterministic' part of a stochastic system it is required to separate the 'deterministic' components of a signal 'buried' in additive noise. Then we need to reconstruct the signal without noise components. Moreover, when dealing with non-stationary processes, the use of a timefrequency representation of a signal is suggested because the produced signals do not lend themselves well to decomposition into sinusoidal components, and they cannot be represented in a meaningful way by Fourier expansions [8]. Therefore, for non-stationary signals, in which frequency value changes at any 
moment, it is more useful to characterise the signal in terms of its Instantaneous Frequency, which is a time dependent representation of the frequency of a signal at any moment. It is the instantaneous frequency which will provide us with information on the presence of a nonlinearity in the signal at any given time instant. In this paper, we present an algorithm for detecting abrupt changes in time series data of van der Pol system with additive white noise by combining the advantages of Discrete Wavelet Decomposition technique, Wavelet energy entropy, and Normalized Hilbert Transform, for computing the instantaneous frequency. The introduction of the wavelet decomposition resolved one key obstacle for computing a meaningful instantaneous frequency from a multicomponent signal by reducing it to a collection of mono-component functions. Once we obtain the mono-component signals, the instantaneous frequency can be computed using the normalized Hilbert transform method.

The rest of the paper is organized as follows: Sec. 2 introduces the van der Pol oscillator with additive noise, whereas Sec. 3 discuses the framework of the discrete wavelet decomposition. Sec. 4 illustrates the details of the proposed approach for estimating the instantaneous frequency peaks. Then in Sec. 5, we present the detection of abrupt changes in simulated data sets produced by the model system. Finally, Sec. 6 concludes the paper with suggestions for future work.

\section{Van der Pol oscillator with additive noise}

This system was initially proposed by van der Pol [9]. It has played an important role in the understanding of nonlinear dynamics in general. Here, we consider a stochastic version with additive white Gaussian noise $\zeta(t)$. The van der Pol equation is an ordinary differential equation describing self-sustaining oscillations in which energy is fed into small oscillations and removed from large oscillations. The van der Pol oscillator can be modelled in its two-dimensional form as:

$$
\begin{aligned}
& \dot{x_{1}}=x_{2} \\
& \dot{x_{2}}=\mu\left(1-x_{1}^{2}\right) x_{2}-x_{1}+\sigma \zeta(t)
\end{aligned}
$$

The position $x_{1}$, and the velocity $x_{2}$ are now random variables. $\mu$ is a scalar parameter indicating the nonlinearity and the strength of the damping. $\sigma$ is the white noise intensity. The Euler scheme is the most widely used approach with noise intensity $\sigma=1.0$ to describe stochastic systems with purely additive noise under standard assumptions. It was shown in [10] that stochastic differential equations of the form

$$
\dot{x}(t)=f(x(t))+\sigma \zeta(t)
$$




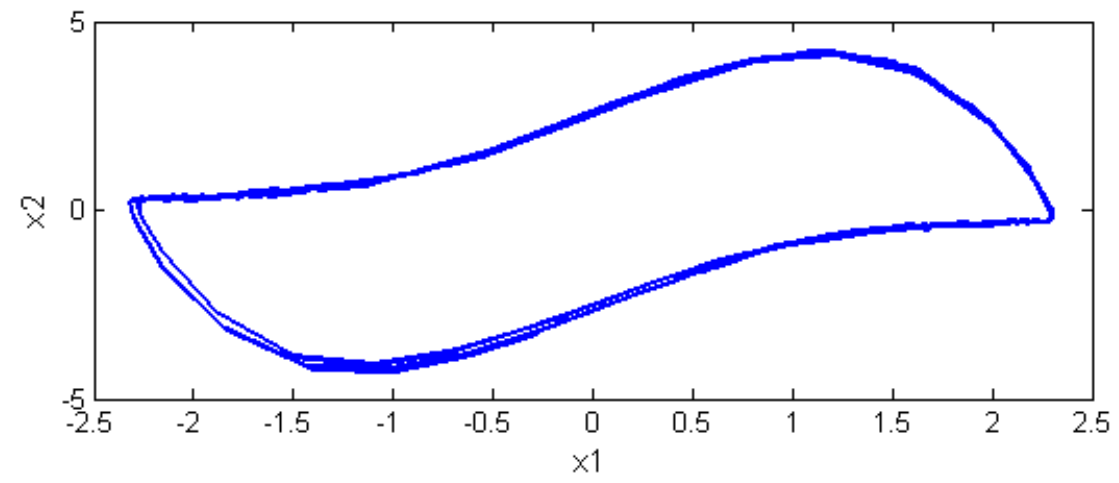

Fig. 1 A sample path of van der Pol oscillator with noise.

can be approximated by a discrete system

$$
x_{n+1}=x_{n}+f\left(x_{n}\right) h+\sigma W_{n} \sqrt{h},
$$

where $h$ is sufficiently small step size, and $W_{n}$ is the standard Wiener process. Above numerical scheme was used to generate time series trajectories of the system (1) and (2). The standard Wiener process is approximated numerically at each step $t_{n}$ by a function that generates psudo-random numbers with expected value $\eta=E_{x}[X]=0$ and standard deviation $\sigma=\sqrt{E_{x}\left[(X-\eta)^{2}\right]}=1$, with $E_{x}$ and $\eta$ denote the expected value and the mean of the process respectively, and $X$ is a random variable.

We are interested here in the asymptotic dynamics of the system. We consider parameter values for which a limit cycle attractor exists in the system in the absence of noise. In the presence of noise the system evolution will lie in the region where there exists the limit cycle attractor. A sample path of our system is presented in Fig. 1. Note that the sample path follows the underlying limit cycle attractor. The parameter values are: $\mu=2$, step size $h=0.1$, and the noise intensity $\sigma=1.0$.

\section{Wavelets and filter banks}

The purpose of this section is to illustrate the connection between filter banks and wavelets, which can be used for analysing the time series data produced by our model system (1) and (2). Wavelets refer to sets of functions of the form

$$
\psi_{(a, b)}(x)=|a|^{-1 / 2} \psi\left(\frac{x-b}{a}\right),
$$

where these sets of functions are formed by dilations and translations of a single function $\psi(x)$. The dilation and translation parameters $a, b$ may range over either a continuous or discrete set. The corresponding set of functions $\psi_{a, b}$ has sufficient members to allow any function $f$ in $L^{2}$ to be reconstructed 


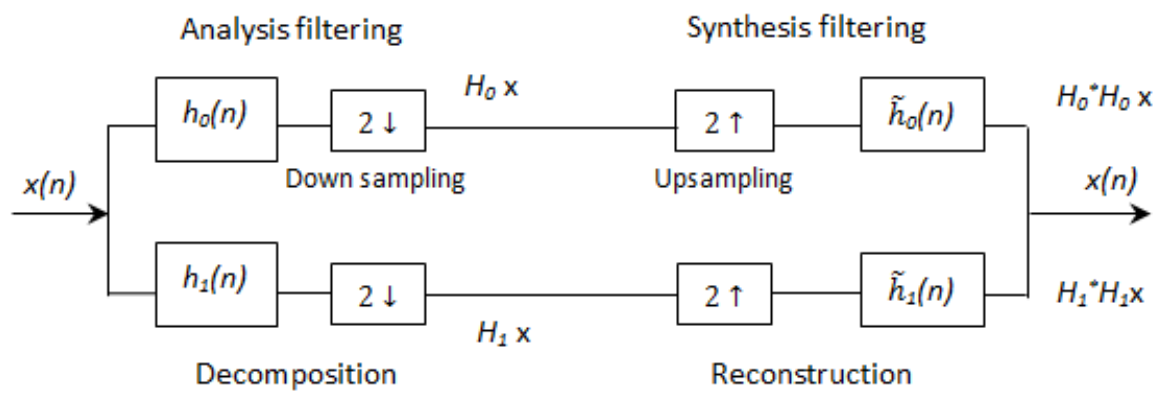

Fig. 2 Orthogonal two channel discrete-time filter bank with analysis filters $h_{0}(n)$ and $h_{1}(n)$ and synthesis filters $\tilde{h}_{0}(n)$ and $\tilde{h}_{1}(n)$.

from its wavelet coefficients $\left\langle f, \psi_{a, b}\right\rangle$ where \langle\rangle denotes the standard $L^{2}$ inner product. The wavelet transform decomposes a function into a weighted sum of its various frequency components. It is useful to decompose the data into wavelet coefficients by using discrete wavelet transform, because most of the coefficients will be close to zero, with only a few coefficients carrying most of the information. Although there are many ways of respecting the $(a, b)$ to a discrete subset of $R$, the most common choice is $a=2^{-k}$, $b=a n$ where $k, n \in Z$. The corresponding discrete wavelet can then be parametrized by a pair of integers $k, n$ rather than $a, b$ as follows:

$$
\psi_{(k, n)}(x)=2^{-k / 2} \psi\left(2^{-k} x-n\right)
$$

We perform discrete wavelet decomposition of a time series data based on the theory of orthogonal filter banks described in [11]. Consider a subband analysis/synthesis filtering system shown in Fig. 2. Analysis stands for splitting a signal into frequency bands, while synthesis stands for reconstructing a signal from various frequency components. Consider real or complex sequences in $l^{2}(Z), x(n), n \in Z$. The inner product is defined as $\langle a(n), b(n)\rangle=\sum a^{*}(n) b(n)$. By convolving $x(n)$ with a filter having impulse response $h(n)$, the output $Y(z)$ is equal to $H(z) X(z)$. Specifically, we want to derive two sub-sampled sequences by filtering with $H_{0}(z)$ and $H_{1}(z)$ and dropping all samples having odd indices. Reconstruction is achieved by resampling at the original sampling rate( replacing the drop samples by zero), filtering with $G_{0}(z)$ and $G_{1}(z)$ and summing up, where $G_{0}(z)=\tilde{H}_{0}(z)$ and $G_{1}(z)=\tilde{H}_{1}(z)$. Therefore, an orthogonal filter bank computes a discrete time wavelet transform when iterated on the octave-band.

\section{Detection of abrupt changes}

The detection process starts by performing a multilevel stationary wavelet decomposition using a specific biorthogonal wavelet $d b 4$ basis function described in [12]. It should be noted that in order to isolate signal nonlinearities, it is more efficient to use a very short basis function. The decomposition process splits the time series data into two parts by using a two-channel filter bank. After splitting, we obtain a vector of 
approximation coefficients $A_{i+1}$ and a vector of detail coefficients $D_{i+1}$, both at different scales, where $i$ is the decomposition level. Then the next step consists of splitting the new approximation coefficient vector $A_{i+1}$ into two parts to obtain a new approximation vector $A_{i+2}$, and details vector $D_{i+2}$. This process continues till we build the wavelet decomposition tree with a number of approximation and details vectors $A_{i+n}$ and $D_{i+n}$, respectively, where $n$ is the number of decomposition levels. Note that, at each stage of the filtering process, the approximation vector is obtained by using a low pass filter to extract the low frequency components while the details vector is obtained by using a high pass filter to extract the high frequency components. The details and approximation coefficients vectors are reconstructed at each decomposition level by using the same filtering approach combined with up-sampling by a factor of 2 .

The obtained wavelet coefficients were placed in a transformation matrix and ordered using two patterns, one contained the smoothed data (approximation coefficients), and the other brought out the details information (details coefficients). It was found that the first few levels contained highest frequencies that mostly consisted of noise, while higher levels contained the basic response of the system. All of these components therefore should be discarded and only the levels that carry most of information of the signal have to be considered for further analysis. Consequently, we have used an energy entropy based method for choosing the right decomposition level to be analysed further. Entropy measures the repeatability or predictability within a time series to quantify the complexity of a signal produced by a system [13]. In our approach, the energy $E$ of each level at time $k$, and for scale $j$ can be approximated by

$$
E(j, k)=\left|s_{i}(t)\right|^{2}
$$

where $s_{i}(t)$ is the reconstructed wavelet coefficients at level $i$. Summing this energy for all discrete time $k$ leads to an approximation of the energy content at each scale $j$ such as $E(j)=\sum_{k} E(j, k)$. Then, we have followed the Shannon entropy, which is a probability density function $P_{E}$ defined as a ratio between the energy of each level and the total energy, that is

$$
P_{E}(j)=\frac{E(j)}{\sum j E(j)} .
$$

This corresponds exactly to the probability density distribution of energy across the scales. Then, we calculate the value of energy entropy $E_{\text {ent }}$ for each decomposition level $i$, which computes the variation of the degrees of complexity of noise such as: 
The larger the value of $E_{\text {ent }}$, the more complicated the signal is. The selection of the decomposition level to be analysed was based on the difference of energy distribution between the noisy time series data at each level. Hence, the value of entropy $E_{\text {ent }}$ at each level $i$ is calculated. The highest difference value of $E_{\text {ent }}$ between two consecutive levels is determined. Then, the higher decomposition level is chosen for further analysis. The selected signal should have higher degree of regularity and contains low noise influences.

The next step of the algorithm process is to apply Hilbert transform to produce an envelop to the identified signal. This envelope is used to normalize the data, and can be computed by Hilbert's equation defined as:

$$
H[s(t)]=p . v \cdot \int_{-\infty}^{\infty} \frac{s(t-\tau)}{\pi \tau} d \tau,
$$

where p.v. denotes the Cauchy principal value of integral [14]. The envelop can be produced by computing the absolute value of Hilbert transform of the signal $s(t)$. This envelop $e(t)$ is used as the base for normalizing the data as follows

$$
n(t)=\frac{s(t)}{e(t)}
$$

where $n(t)$ is the normalized data. Ideally, $n(t)$ should have all the extrema with unity value. Such normalization is particularly important because it enables us to compute the phase angels directly without any approximation. The next step is to apply Hilbert transform to the normalized data to obtain an analytic complex signal such as

$$
H[n(t)]=x(t)+j y(t)=a(t) e^{j \phi(t)},
$$

where $a(t)$ is an absolute value of the signal, and $\phi(t)$ is a phase function. The imaginary part includes phase information that depend on the phase of the original data. Once the phase angles are determined, the instantaneous frequency $f_{s}$ can be computed as a derivative of the phase function $\phi(t)$ as follows:

$$
f_{s}(t)=\frac{1}{2 \pi} \frac{d \phi(t)}{d t}
$$



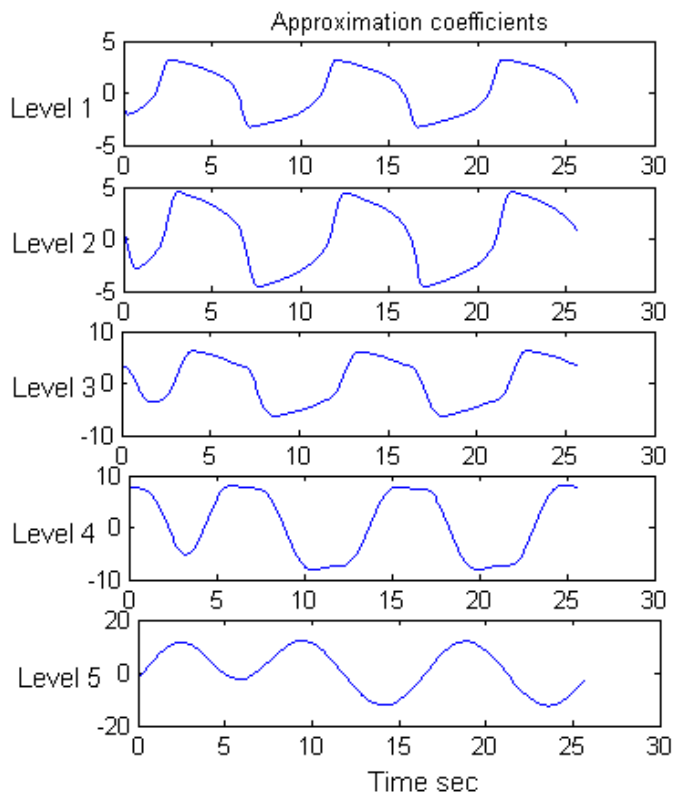
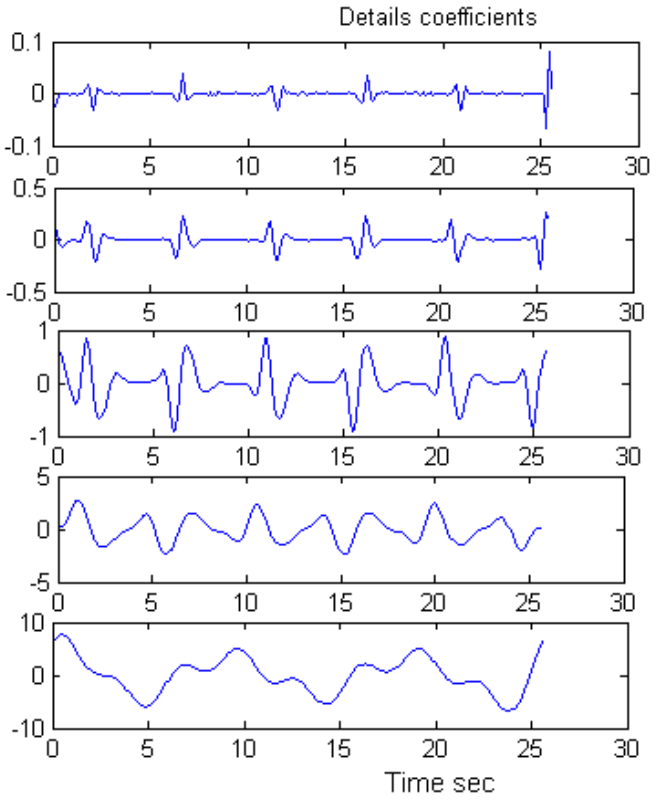

Fig. 3 Wavelet approximation coefficients (left column), and wavelet details coefficients (right column).

This indicates that if $f_{s}$ is a function of $t, f_{s}(t)$ provides a measure of the frequency domain signal energy concentration as a function of time. The strongest peaks, in the time-frequency representation, allowed us to identify any abrupt changes in the time series data.

\section{Numerical experiments}

In this section, we apply the detection process to identify abrupt changes in the signal produced by our model system (1) and (2) with noise intensity $\sigma=1.0$. For the purpose of our analysis we use the position data. We consider the length of the time series required to make three revolutions around the underlying limit cycle attractor (the limit cycle attractor is present in the system in the absence of noise). The total analysis time is $26.5 \mathrm{~s}$. The discrete wavelet transform was applied to decompose the data into five levels as shown in Fig. 3. The wavelet decomposition technique brings out high resolution data which are the details coefficients, while at the same time it extracts the remaining data as approximation coefficients. The selection of the decomposition level to be analysed was based on the calculated $E_{\text {ent }}$ values of the noisy data at each decompisition level. As shown in Fig. 4, the highest entropy difference is between levels 2 and 3 . Hence, level 3 was selected for further analysis. The reconstruction process was applied to the signal of this level to produce a reconstructed data shown in Fig. 5.

The frequency analysis method was then applied to the reconstructed data where we can isolate the presence of harmonics, thereby confirming the presence of the limit cycle attractors. We start the normalization process by calculating Hilbert's envelop to the signal. Fig. 6 shows a comparison between 


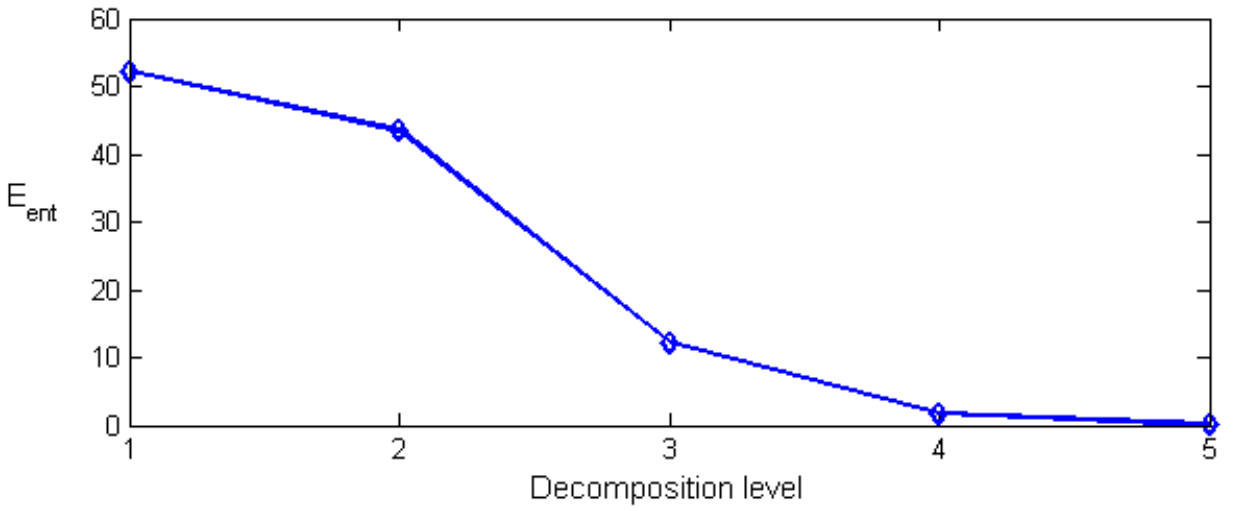

Fig. 4 Wavelet entropy energy of the time series data at each decomposition level.

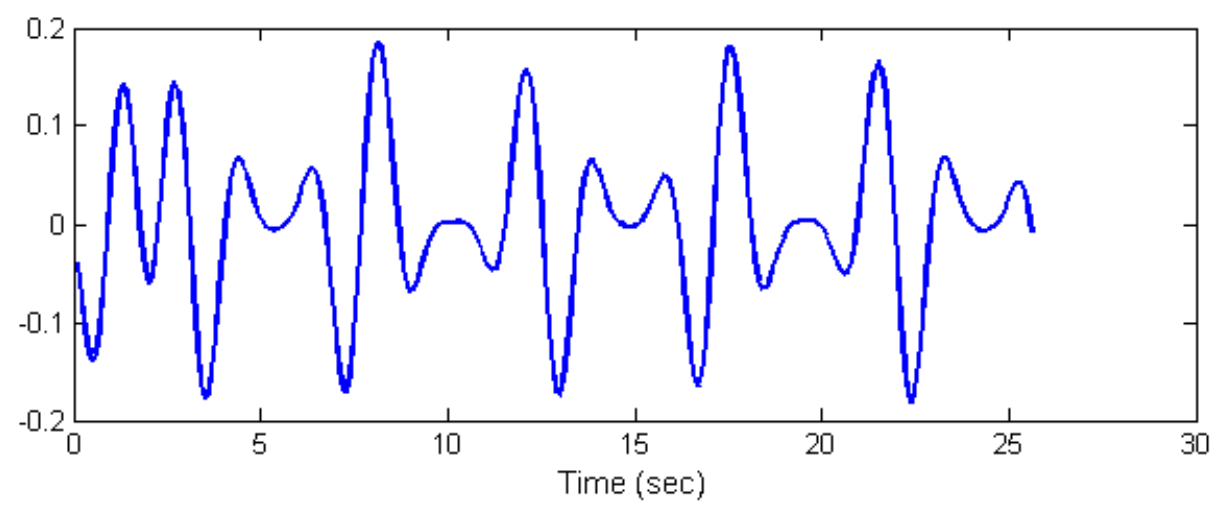

Fig. 5 The reconstructed wavelet coefficients associated with level 3.

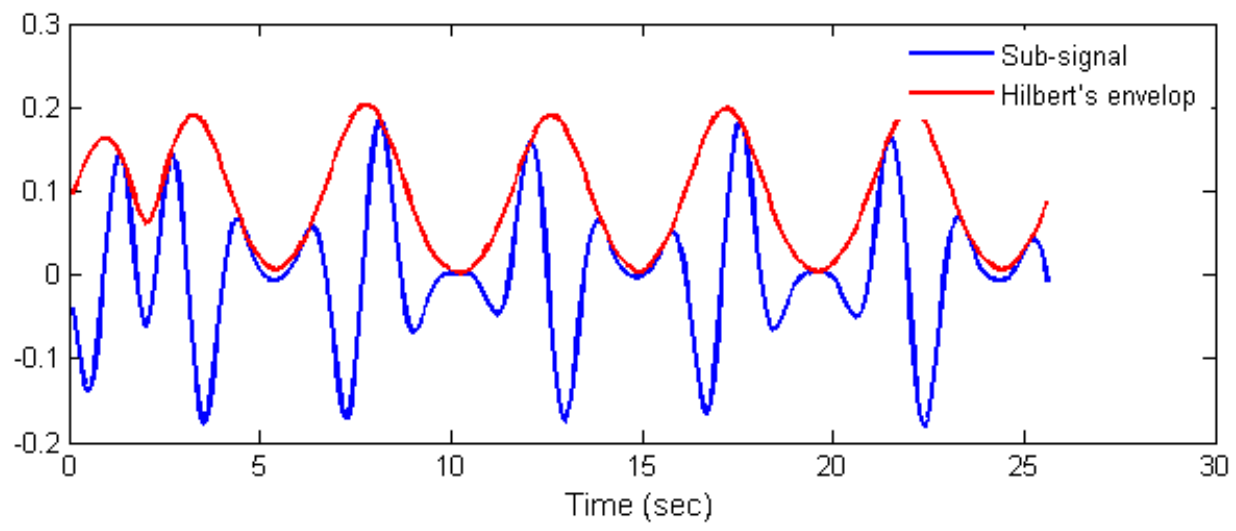

Fig. 6 The identified signal with Hilbert's envelop.

the obtained data and the calculated Hilbert's envelop. Fig. 7 presents the produced signal after the normalization scheme where all of the values of the signal are less than or equal to unity. This normalized carrier enables us to provide a ready and sharper local energy based measure of any abrupt changes. The instantaneous frequency of the data can then be computed by applying Hilbert transform. This approach will enable us to get an exact instantaneous frequency as a derivative of the phase function. Fig. 8 shows the time-frequency presentation of the time series data generated by our stochastic model system. 


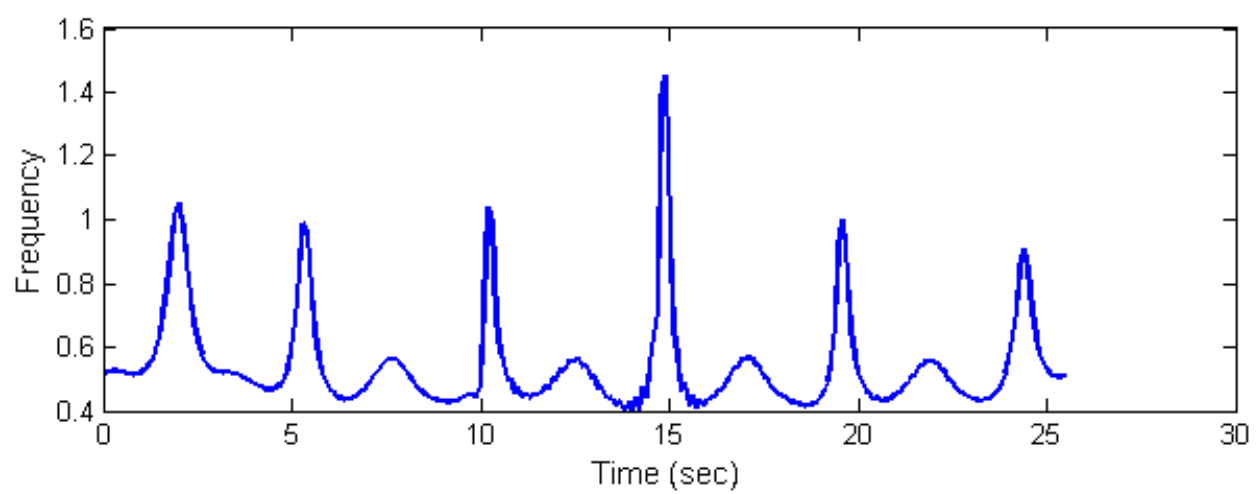

Fig. 8 Time-frequency presentation of the simulated data.

It can be seen in Fig. 8 that the proposed approach accurately locates the change points in the time series data. Note that, as shown in Fig. 1 the sample trajectory has two abrupt turning points when crossing the $\mathrm{x}$-axis. Since the sample trajectory crosses the $\mathrm{x}$-axis six times, we expect to find six time instances where the sought abrupt changes occur. Six peaks were identified in the time-frequency representation by means of our algorithm. These changes occur at $t=2.0 \mathrm{~s}, t=5.3 \mathrm{~s}, t=10.2 \mathrm{~s}, t=14.9 \mathrm{~s}$ $t=19.6 \mathrm{~s}$, and $t=24.4 \mathrm{~s}$. This suggests that the instantaneous frequency is able to capture the change points produced by the stochastic system.

We further investigate the effect of additive white noise on detecting abrupt changes in the time series data produced by our model system (1) and (2) with different noise intensity levels. We set $\sigma$ equal to 1.3 , 2, 5, and 30. Fig. 9 shows evolution of sample paths for the four different cases. It can be seen that adding higher noise intensity significantly increases the difficulty of detecting change points in system dynamics. In the first two cases when $\sigma=1.3$ and 2.0, all the six frequency peaks are successfully identified. The abrupt changes occur approximately at the same location as in the test case $(\sigma=1.0)$. By analysing the third and fourth case, the algorithm was also able to identify the change points. However, we found that there are some missing instantaneous frequency peaks. We conjecture that high noise intensity has 


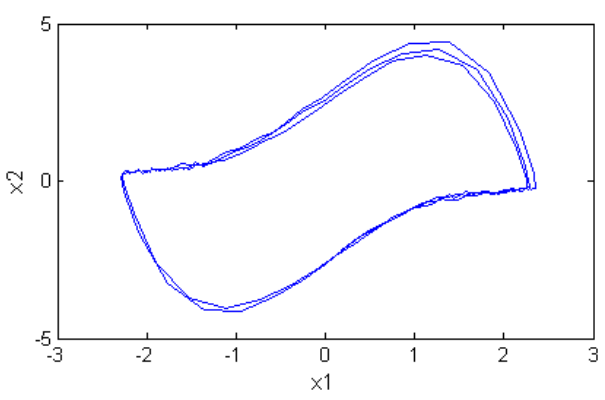

(a) $\sigma=1.3$

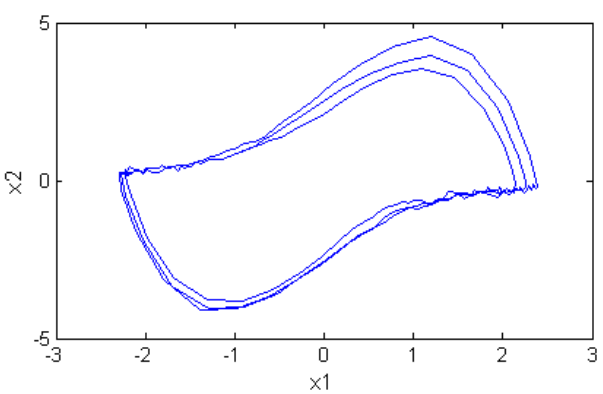

(c) $\sigma=2.0$

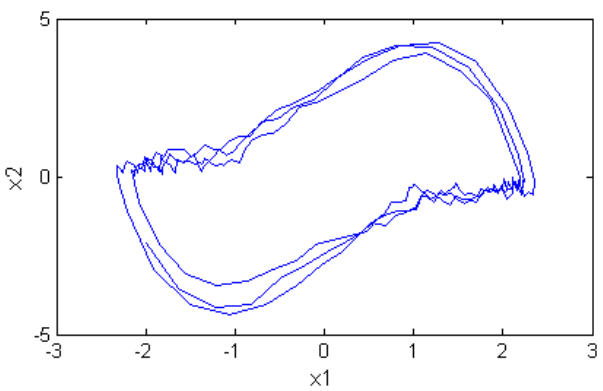

(e) $\sigma=5.0$

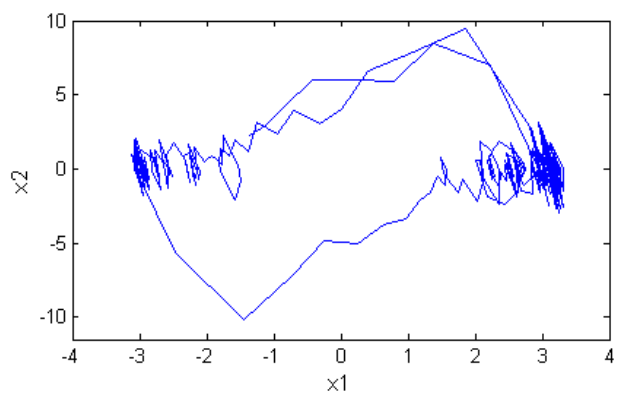

(g) $\sigma=30.0$

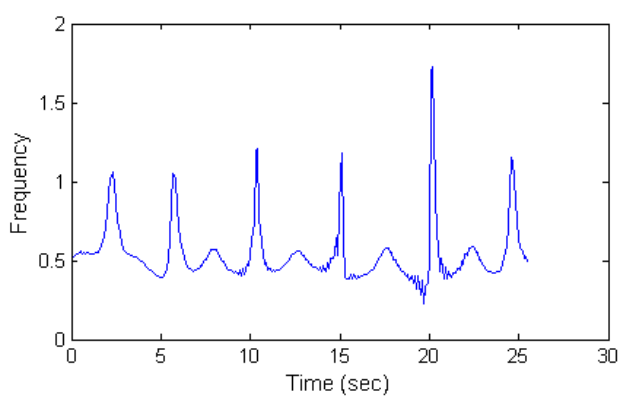

(b) $\sigma=1.3$

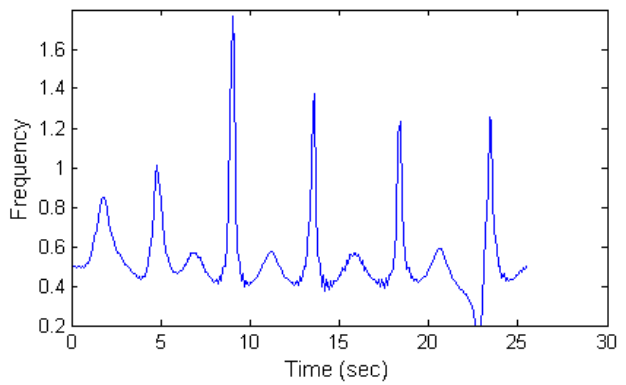

(d) $\sigma=2.0$

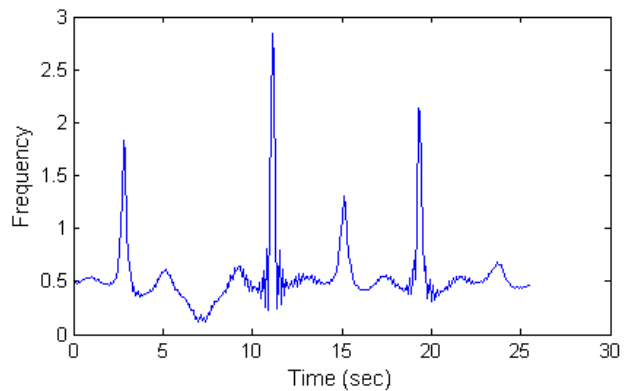

(f) $\sigma=5.0$

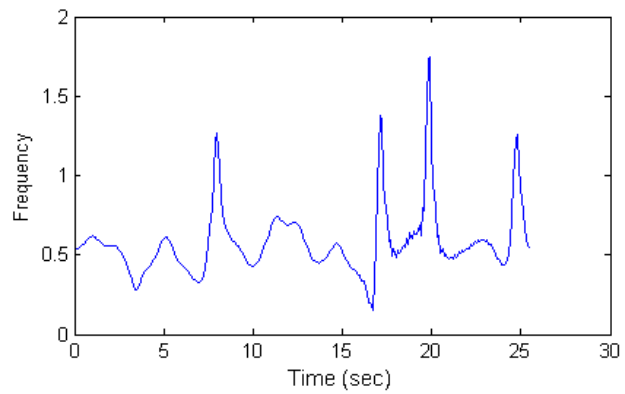

(h) $\sigma=30.0$

Fig. 9 Subfigures (a,c,e,g) show evolution of the limit cycles in the phase plane for the model system (1) and (2) with different noise intensity $\sigma$. Subfigures $(\mathrm{b}, \mathrm{d}, \mathrm{f}, \mathrm{h})$ show the corresponding instantaneous frequency peaks of the stochastic model system (1) and (2) for different noise intensity $\sigma$. 
a linearising effect on the underlying nonlinearity present in the system, and it is the nonlinearity which creates the abrupt turning points in system evolution. There is also a slight shift in the identified peaks as a result of the influence of noise on the system. Hence, the noise intensity should not exceed a certain system dependent threshold level in order to detect such abrupt changes in the evolution.

\section{Conclusion}

In this article, we propose a method based on wavelet and frequency analysis for detecting abrupt changes in the process data of the van der Pol oscillator model with noise. The advantage of this technique is that it allows us to decompose the data into a set of independent coefficients (mono-components) corresponding to orthogonal basis functions. These mono-components are then analysed and recombined into a signal that contains the instantaneous frequency reflections, but not the system main response or noise. The output is a series of peaks, each of which occurs when the system trajectory exhibits abrupt change. The effectiveness of the developed algorithm in detecting such changes arises from the fact that abrupt changes manifest themselves as spikes in the time-frequency plane. Thus, the proposed method is promising in detecting not only features such abrupt changes but also discontinuities in time series data. Further work is aimed at investigating the algorithm's ability to locate change points in the data generated by switched control systems with noise. The discontinuities which we seek to identify in such systems occur due to switchings between differentiable vector fields.

\section{References}

1. Mandal, P.S., Banerjee, M.: Deterministic and Stochastic Dynamics of a Competitive Phytoplankton Model with Allelopathy. Differential Equations and Dynamical Systems 21, 341-372 (2013).

2. Arnolda, L., Imkellerb, P., Wu, Y.: Reduction of deterministic coupled atmosphereocean models to stochastic ocean models: a numerical case study of the LorenzMaas system. Dynamical Systems: An International Journal 4(18), 295-350 (2003).

3. Lian, J., Zhang, F., Shi, P.: Sliding mode control of uncertain stochastic hybrid delay systems with average dwell time. Circuits Syst. Signal Process 31, 539-553 (2012)

4. Sharma, S.N.: A Kolmogorov-Fokker-Planck approach for a stochastic Duffing-van der Pol system. Differential Equations and Dynamical Systems 4(16), 351-377 (2008).

5. Theron, J., Aldrich, C.: Identification of nonlinearities in dynamic process systems. Journal of the South African Institute of Mining and Metallurgy 104, 191C200 (2004).

6. Babji, S., Gorai, P., Tangirala, A.: Detection and quantification of control valve nonlinearities using hilbert-huang transform. Advances in Adaptive Data Analysis 1, 425-446 (2009).

7. Killick, R., Eckley, I.: A wavelet-based approch for detecting changes in second order structure within nonsatationary time series. Electronic Journal of Statistics 7, 1167-1183 (2013). 
8. Boashash, B.: Estimating and interpreting the instantaneous frequency of a signal. Proceedings of the IEEE 80, 520-538 (1992)

9. van der Pol, B.: On relaxation oscillations. Phil. Mag. 2(11), 978-992 (1926).

10. Kloeden, P.E., Platen, E.: Numerical Solution of Stochastic Differential Equations. Springer-Verlag (1995).

11. Strang, G., Nguyen, T.: Wavelets and filter banks. Wellesley Cambridge Press (1997).

12. Daubechies, I.: Ten lectures on wavelets. CBMS-NSF Series in Applied Mathmatics. SIAM, Philadelphia 61 (1992).

13. Richman, J.S., Moorman, J.R.: Physiological time-series analysis using approximate entropy and sample entropy. Am J Physiol Heart Circ Physiol. 278, H2039-H2049 (2000).

14. Marple, S.: Computing the discrete-time analytic signal via fft. IEEE Transactions on Signal Processing 47, 52,600-2603 (1999). 
Copyright Transfer Statement
Click here to download Copyright Transfer Statement: 12591_CTS_DEDS_08062010.pdf

Copyright Transfer Statement
Click here to download Copyright Transfer Statement: 12591_CTS_DEDS_08062010.pdf (1) 